'llu. Revista de Ciencias de las Religiones

ISSN: 1135-4712

http://dx.doi.org/10.5209/ILUR.57413

\title{
Tras las huellas de lo sagrado: un repaso crítico por las propuestas darwinistas para explicar la conducta religiosa
}

\author{
Álvaro Gómez Peña ${ }^{1}$
}

Recibido: 12 de diciembre de 2016 / Aceptado: 6 de febrero de 2017

Resumen. En el presente estudio se realiza un repaso historiográfico por las principales hipótesis que se han propuesto desde la epistemología darwinista para explicar la conducta religiosa. Para ello, en primer lugar se analizan los pilares básicos del darwinismo: variación, herencia y selección. Teniendo presente esta tríada de conceptos, en un segundo punto se pasa a examinar el fenómeno religioso desde dichos presupuestos teóricos. En este sentido, los darwinistas han analizado la religión bien como una conducta neutra en tanto que exaptación, como un fenómeno no-adaptativo desde una perspectiva memética en tanto que anacronismo y como una práctica adaptativa desde un punto de vista individual y multinivel. En último lugar, se aporta una reflexión crítica sobre los problemas y soluciones que aporta cada perspectiva.

Palabras clave: Historiografía; Exaptación; Memética; Selección multinivel.

\section{[en] Following the traces of the sacred: a critical review of Darwinian proposals to explain religious behaviour}

\begin{abstract}
In the present study a historiographical review is performed according to the main hypotheses have been proposed from a Darwinian point of view to analyse religion. First, the basic pillars of Darwinian theory are analysed: variation, inheritance and selection. Keeping in mind these previous ideas, Darwinists theorists have analysed the religion well as neutral phenomenon while exaptation, as a non-adaptive phenomenon from a memetic perspective while anachronism and as an adaptive phenomenon from a single and multilevel standpoint. Finally, a critical review is realised about problems and solutions of each perspective.

Keywords: Historiography; Exaptation; Memetics; Multilevel Selection.
\end{abstract}

Sumario. 1. Introducción 2. Análisis darwinista del fenómeno religioso 2. 1. La religión como fenómeno neutro: subproducto 2.2. La religión como fenómeno no-adaptativo: memética y anacronismo. 2.3. La religión como fenómeno adaptativo: individual y multinivel 3. Análisis crítico a modo de conclusión. 4. Bibliografía.

Cómo citar: Gómez Peña, A. (2017), Tras las huellas de lo sagrado: un repaso crítico por las propuestas darwinistas para explicar la conducta religiosa, en 'Ilu. Revista de Ciencias de las Religiones 22, 203220.

\footnotetext{
1 Universidad de Sevilla (España).

E-mail: agomez19@us.es
} 


\section{Introducción}

Tradicionalmente ha existido un intenso debate entre los defensores del evolucionismo darwinista y los partidarios del creacionismo veterotestamentario sobre la idoneidad de una $\mathrm{u}$ otra corriente para explicar la generación y existencia de la vida en la naturaleza. Dicha controversia se ha venido dando desde la publicación en 1859 de El origen de las especies por parte de Charles Darwin. En este sentido, famoso fue y sigue siendo el debate de Oxford que tuvo lugar en 1860 entre Samuel Wilberforce, obispo de dicha localidad, y Thomas Henry Huxley, biólogo británico.

Siglo y medio después de la publicación de la más famosa obra de Darwin, dicha controversia sigue estando latente entre buena parte de la población. Sirva como ejemplo la encuesta elaborada por la empresa Gallup entre individuos adultos de Estados Unidos aprovechando que se cumplían 150 años de la aparición de El origen de las especies. ${ }^{2}$ En ella, el porcentaje de encuestados que está a favor de la teoría de la evolución crece si su nivel de estudios es mayor, lo que demuestra que las instituciones educativas juegan un importante papel en este asunto. Por otra parte, existe una relación inversa entre la regularidad con la que los encuestados asisten a ceremonias religiosas y el ser partidario de la evolución darwinista.

La respuesta a porqué el darwinismo y la religión aparecen con frecuencia como modelos incompatibles reside en que la inmensa mayoría de los credos entienden que las especies son inmutables, o a lo sumo transformistas -que han ido cambiando su aspecto pero no han dejado de ser esas especies y no otras-, lo que se ha venido definiendo tradicionalmente como una visión esencialista de la cuestión. A este respecto, sin lugar a dudas existen características distintivas de cada ser vivo que le hacen único, como la combinación de su color de ojos, sus marcas en la piel, su tipo de pelo, su estatura, su complexión, etc. Sin embargo, desde la postura esencialista estas características son accidentales. Así, desde el principio de los tiempos han existido las mismas especies - esencias- como si de compartimentos estancos se tratara, y aunque dichas diferencias -accidentes- entre seres de una misma especie sean notables, de ningún modo pueden trascender dichos compartimentos estancos.

Rompiendo con esta forma de pensamiento, Darwin se aupó sobre los hombros de quienes antes que él habían propuesto eliminar el concepto fijista y otorgar todo el protagonismo a los accidentes individuales con su teoría de la selección. Es decir, a la suma de características propias de cada individuo que se irían transmitiendo de generación en generación con modificaciones -lo que se conoce hoy como genes y Darwin intuyó como gémulas-. Sería por tanto la suma de esas modificaciones genéticas a lo largo de miles y miles de años la que habría conseguido potenciar especies tan disímiles entre sí hasta el punto de que si se intentaran cruzar entre ellas no dejarían descendencia fértil.

Hecha esta aclaración, el presente estudio no pretende dar cuenta de los enfrentamientos entre darwinistas y fijistas. Lo que se quiere en estas páginas es mostrar que a pesar de dicha aparente confrontación, es posible analizar la conducta religiosa desde una óptica darwinista. Se trata de una cuestión en absoluto carente de

http://www.gallup.com/poll/114544/darwin-birthday-believe-evolution.aspx [Web consultada por última vez el 11 de marzo de 2017]. 
dificultad, pues la especificidad que ha caracterizado al ser humano en este ámbito es el principal motivo por el cual Edward O. Wilson, el padre de la sociobiología, llegó a calificar el estudio de la religión como el mayor desafío al que se enfrenta el darwinismo. ${ }^{3}$

Por conducta religiosa se entiende la creencia en una o varias fuerzas sobrenaturales, es decir con mayores capacidades que las que veríamos en la naturaleza sin su concurso, que pueden influir positiva y/o negativamente en las vidas de sus fieles, para lo cual se establece una relación con dichas fuerzas personificadas o no, mediante rituales y tabúes que intentan incentivar los comportamientos positivos y minimizar los negativos. Vista la religión desde esta perspectiva, desprovista de cualquier etiqueta que pueda nublar la comprensión del discurso, los investigadores darwinistas han tratado de explicar el fenómeno religioso del mismo modo que habrían abordado cualquier otro tema.

\section{Análisis darwinista del fenómeno religioso}

Nadie duda de que la evolución -no el darwinismo- sea un hecho. Existe una gran cantidad de datos mensurables que avalan esta realidad a distintas escalas. En este sentido, el estudio del pasado, humano o no humano, no escapa a ello. Sin embargo, explicar cómo suceden los cambios no es tarea fácil ni cuenta con una unanimidad de criterios entre los investigadores que han abordado esta cuestión. ${ }^{4}$

La propuesta que cuenta con el respaldo de la mayoría de los científicos es la corriente darwinista. El motivo de tal apoyo se encuentra, siguiendo a E. Mayr, ${ }^{5}$ en el hecho de que este cuerpo epistemológico ha sabido conjugar cinco grandes ideas complementarias para explicar la historia de la vida sobre la Tierra: (1) el cambio perpetuo, que establece que la naturaleza está cambiando continuamente; (2) el origen común, que postula que cada grupo de organismos procede de un antecesor común, y en consecuencia, existe un origen único para todos los seres vivos; (3) la diversificación de las especies, que explica cómo se produce la enorme diversidad de los seres vivos; (4) el gradualismo, que propone cambios paulatinos en las poblaciones para la aparición de nuevas especies; y (5) la selección natural, un mecanismo de reproducción diferencial que favorece a los mejor adaptados al entorno.

Esta última idea - la selección natural- se basa en tres pilares básicos. Primero, debe existir un registro sobre el que quepa la posibilidad de copia-herencia-. En segundo lugar, la herencia de dichos rasgos no puede ser una réplica perfecta sino abierta a mutaciones o modificaciones de un individuo a otro, lo que origina variación en el registro. En último lugar, deben existir diferencias en la aptitud del registro, es decir las interacciones entre el registro y el medio ambiente en el que existe el número de copias será disímil dada su variación, lo que comúnmente se denomina como selección. En consecuencia, el número de ejemplares con éxito para la siguiente reproducción se habrá visto de nuevo sometido al mismo proceso -feedback-. En esencia, estos tres sencillos requisitos son los pilares básicos para poder

Wilson 1980, 246.

vid. Gutiérrez 2010, 15-16.

Mayr 1992, 48-50. 
articular una explicación darwinista de la vida. ${ }^{6}$ Sin embargo, las implicaciones de estos tres puntos van más allá.

Teniendo muy presente estos elementos, la escuela darwinista se caracteriza por determinar cuáles son dichos rasgos heredables, cómo ocurre dicho proceso y las causas y consecuencias que se derivan de él. Es esta la metodología que se ha utilizado para tratar de explicar la conducta religiosa.

Desde la perspectiva de la tríada darwinista herencia-variación-selección, la existencia de la conducta religiosa ha sido enfocada desde las tres perspectivas posibles en que toda mutación es estudiada por los darwinistas. A saber, que la religión sea un comportamiento adaptativo, negativo o neutro. Esta cuestión que aquí se trata mantiene dividido a un buen número de psicólogos y antropólogos darwinistas. De una parte, se encuentran aquéllos que consideran que la religión ha sido adaptativa en términos de descendencia. Es decir, que los individuos o los grupos religiosos dejaron mayor número de descendientes y por lo tanto pudieron transmitir esta conducta de generación en generación a más personas en detrimento de quienes no lo eran. No hay que confundir el término adaptativo con cuestiones de otra índole que no sean estrictamente reproductivas, que es como los darwinistas interpretan el éxito evolutivo de una mutación, desprovisto de cualquier connotación social, política o económica. De otra parte, se encuentran quienes opinan que la religión influye negativamente en el éxito reproductivo de nuestra especie. Mientras que hay un tercer grupo de autores que consideran que la religión no ofrece datos ni a favor ni en contra del éxito reproductivo y que por lo tanto es una conducta neutra en los términos aquí tratados. ${ }^{7}$

Por lo general, estas tres perspectivas parecen incompatibles, sin embargo el problema se encuentra en las preguntas de partida que han hecho los investigadores y en varias malinterpretaciones de la teoría darwinista a la hora de dar respuesta a dichos interrogantes. Así, los partidarios de ver la religión como una conducta positiva en términos biológicos se han venido preguntando por qué es adaptativa la religión y cómo se han generado las conductas que lo son. Los que ven los comportamientos religiosos como negativos se preguntan cómo es posible que persistan y se transmitan los conceptos religiosos de individuo a individuo. Mientras los terceros se plantean cuál es el origen cognitivo del pensamiento religioso -en este último caso, aunque los estudios cognitivos son asumidos generalmente por los investigadores darwinistas, los cognitivistas no tienen por qué aceptar los postulados de dicha corriente epistemológica-.

Con base en estas tres posiciones y sus preguntas de partida, se han creado cinco hipótesis de trabajo que la inmensa mayoría de las veces han sido vistas por los interesados de nuevo como incompatibles, cuando no contrapuestas. Sin embargo, un recorrido por ellas y sus implicaciones permite arrojar luz sobre los malentendidos de unos y otros. Quienes ven la religión como un fenómeno que no puede entenderse en términos adaptativos o negativos, sino como una exaptación, se han propuesto verla como un subproducto de otras adaptaciones (1). Para aquellos que

Gould 2004, 25-38.

Cinco buenas aproximaciones a este galimatías teórico y los posibles caminos metodológicos que se han andado desde el darwinismo en su aplicación a la conducta religiosa se encuentran en J. W. Dow 2006, M. Ruse 2008, 304-312, G. Andrade 2009, 305-334 y T. Bourrat 2015. La misma problemática en términos generales ha sido expuesta por L.-G. Pérez-Aguilar 2011. 
defienden que la religión es un fenómeno negativo en términos adaptativos se ha barajado para su explicación una hipótesis memética (2) y una anacrónica (3). En último lugar, los que aceptan que la religión sí es un fenómeno adaptativo desde un punto de vista darwinista han realizado una explicación a nivel individual (4) y otra a escala multinivel (5). ${ }^{8}$

\subsection{La religión como fenómeno neutro: subproducto}

De una parte, se encuentran los investigadores que sostienen que el pensamiento religioso ha surgido como un subproducto (1) de las auténticas conductas y módulos cerebrales que sí son adaptativos. El término subproducto -byproduct- es empleado por los autores que lo utilizan como sinónimo de exaptación. Este concepto puede explicarse del siguiente modo. Dado un rasgo determinado, ya sea de un individuo o de un conjunto de individuos, que evoluciona originalmente como adaptación a unas determinadas condiciones, una vez consolidada su adaptación acaba empleándose con una finalidad diferente de la que tuvo en origen. Un ejemplo terminará de aclarar esta definición. Se ha propuesto que varios dinosaurios no voladores habrían contado con plumas entre sus atributos corporales. Dichas plumas habrían actuado como reclamos sexuales, derivándose en un mayor éxito reproductivo de quienes las portaban. En ningún caso tales plumas acabaron teniendo valor adaptativo como atributos que permitían a dichos dinosaurios volar. Tan sólo con el paso de millones de años las plumas acabaron siendo utilizadas como instrumentos aeronáuticos por parte de las aves, influyendo en su tasa de reproducción. A este cambio de uso se le denomina exaptación.

Siguiendo esta línea argumental, los autores que defienden que la religión sería una exaptación consideran que ésta no ha sido en sí misma una conducta adaptativa. El verdadero rasgo adaptativo habría sido la antropomorfización de la naturaleza y la hiperdetección de agentes en ella. El primero en abrir el camino desde esta perspectiva fue S. Guthrie. ${ }^{9}$ Para este autor, los humanos tenemos tendencia a dar características animadas y antropomorfistas a la naturaleza, por lo que la religión pudo tener su punto de partida en esta expresión. Terremotos, inundaciones, lluvias o erupciones volcánicas, por ejemplo, serían fruto de la intencionalidad de actores invisibles «sobrenaturales» que reflejarían sus actos a través de la naturaleza. En estos términos expresaba su idea en el artículo en que dio por primera vez a conocer su idea:

My argument may be put in five propositions and two informal deductions, as follows:

P1. Phenomena (aspects of the world as known through our senses) initially are ambiguous.

D1. Therefore phenomena (at least salient or potentially significant ones) must be interpreted.

P2. Phenomena are interpreted by a set of models based on experience of analogous phenomena.

Smith y Arrow 2010, 49.

Guthrie 1980; 1993. 
P3. A model by which to interpret a phenomenon in a context is chosen from the set by (a) its capacity to generate the phenomenon, (b) the likelihood of occurrence of the phenomenon from which the model derives, and (c) its subjective importance to the observer.

P4. Humans, (a) because they are complex and multifaceted, generate a very wide range of phenomena; (b) as social beings, are likely to be wherever the human perceiver may be; and (c) are the most important factor in the human environment.

D2. Therefore human-like models frequently are chosen to interpret ambiguous phenomena.

P5. Generalization and systematization of this choice is the cognitive basis of religion. ${ }^{10}$

El posible surgimiento de la religión como una antropomorfización de la naturaleza tiene cumplida respuesta en la teoría de juegos. ${ }^{11}$ Aun así, la fuerza de esta hipótesis radica en que la antropomorfización no es un recurso grupal para explicar el comienzo de las creencias en lo sobrenatural, sino individual y compartido por todos los seres humanos dadas sus características cerebrales. Basado en este supuesto, la religión se construiría a partir de estas percepciones individuales, repitiéndose el proceso en cada persona. Se asume así que el origen de las creencias puede rastrearse antropológicamente en el presente, y no es necesario acudir al pasado para buscarlo.

Al igual que Guthrie, autores como P. Boyer, ${ }^{12} \mathrm{~S}$. Atran ${ }^{13}$ y L. Kirkpatrick ${ }^{14}$ entre otros consideran que nuestro cerebro ha evolucionado desarrollando capacidades como la superdetección de agentes en la naturaleza, o lo que es lo mismo, creer como primer impulso que los sonidos o movimientos que percibimos no son fruto del viento, las hojas o el agua, sino de seres vivos, por lo que se acaban vivificando los hechos. Estos investigadores consideran que esta postura ha sido bastante adaptativa en el pasado, ya que pudo permitir a nuestra especie sobrevivir más fácilmente estando alerta. A ello se suman otras hipótesis de carácter cognitivista, como intentar dar explicación de manera intuitiva a lo que ocurre en la naturaleza, aunque ello conlleve dar respuestas contraintuitivas que entren dentro del plano de lo religioso.

A priori podría ser poco razonable que las ideas contraintuitivas, es decir, aquéllas que parecen contradecir la lógica que vemos en los hechos, sean tenidas en cuenta y transmitidas con mayor éxito que otras. Sin embargo, autores como J. L. Barrett y M. A. Nyhof han demostrado con varios experimentos que lo cierto es lo contrario. El primero de los llevados a cabo consistía en dar a leer a los participantes historias con ideas ordinarias y contraintuitivas. Nada más acabar de leerlas, se les pedía que contaran lo que habían leído. Ambos autores comprobaron que los lectores recordaban un $60,3 \%$ de los conceptos contraintuitivos en las obras frente a un $43,3 \%$ de los conceptos ordinarios. El segundo experimento estuvo estructurado de similar manera, aunque añadiendo conceptos poco usuales, como por ejemplo un periódico rosa, que si bien puede ser posible es altamente improbable de experimentar en la vida cotidiana. Sin embargo, esta vez se pidió a los lectores que contaran la

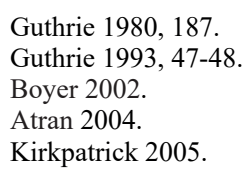


historia a un tercero y éstos a un cuarto hasta alcanzar tres generaciones. Los resultados fueron semejantes. Los conceptos contraintuitivos eran los más transmitidos. El tercer experimento consistió en hacer contar la historia a un tercero. Los resultados mostraban nuevamente que los hechos contraintuitivos eran recordados por encima de los usuales -un $71,1 \%$ frente a un $43,5 \%$ - . La novedad esta vez radicaba en que a los participantes se les pidió recordar la historia tres meses después, siendo los porcentajes de un $57,2 \%$ frente a un $21,0 \%$. De igual modo, el último experimento consistió en sustituir los elementos cotidianos por elementos bizarros. De nuevo los resultados fueron los mismos. La conclusión a la que llegaron Barrett y Nyhof es que los conceptos contraintuitivos son más recordables que los lógicamente posibles, y por tanto su probabilidad de transmisión es mayor. ${ }^{15}$

En relación con estos experimentos, I. Pyysiainen, M. Lindeman y T. Honkela llevaron a cabo otra serie de estudios en que se ponía a prueba si los sucesos contraintuitivos en los que intervenían personajes eran considerados más probablemente religiosos o no. Para tal fin, se transfirieron características humanas a los animales, aspectos psicológicos a objetos naturales o fenómenos, y se le negaron cualidades físicas o biológicas a agentes intencionales. Los sujetos preguntados tenían que responder en una escala de 1 a 5 siendo 5 el nivel de religiosidad más alto. Los resultados de los dos primeros estudios mostraban que los individuos juzgan los sucesos contraintuitivos como más probablemente religiosos que los intuitivos (estudio 1: $M=3.52$ y $M=2.14$; estudio $2: M=3.46$ y $M=1.80$ ). Los resultados para el tercer caso determinaron que no sólo los sucesos contraintuitivos eran juzgados como más religiosos, sino que los sucesos contraintuitivos que tenían agentes implicados eran considerados del mismo modo $(M=3.39)$ frente a los que no $(M=2.47)$. Al igual que los sucesos contraintuitivos en los que un agente tenía en cuenta los asuntos humanos eran vistos como más religiosos $(M=3.57)$ que aquéllos en los que el agente era inconsciente $(M=3.12){ }^{16}$

Al igual que las capacidades contraintuitivas, existen muchos mecanismos que han sido explicados por los seguidores de la hipótesis de la religión como subproducto. Son numerosas las sociedades que muestran una recurrencia en sus religiones a creer en la vida más allá de la muerte, en seres con poderes especiales, en los milagros y las señales sobrenaturales, en el creacionismo, en la posesión de los espíritus, en la necesidad de realizar rituales o en el castigo divino. Algunas de estas cuestiones han sido tratadas por los psicólogos y antropólogos darwinistas, como la creencia en el más allá en términos de nuestra incapacidad para simular la eliminación de nuestros estados mentales o las nociones de seres con poderes especiales como forma de explicar cuestiones que no encajan con la lógica que percibimos en la naturaleza, o el creacionismo como una predisposición a razonar de manera teleológica buscando una intencionalidad en las cosas que hacemos y ocurren a nuestro alrededor. Sin embargo, los autores partidarios de ver la religión como un subproducto han asumido que su postura es incompatible con la visión adaptativa de la religión. Es aquí donde se malinterpreta el sentido adaptacionista que propone el darwinismo. Cuando los humanos desarrollaron los módulos cerebrales que intervienen en la conducta religiosa, no lo hicieron con ninguna finalidad previa. Las 
mutaciones genéticas que desarrollaron dichos módulos se producen por azar. No hay teleología en ello.

Vistas así las cosas, muy probablemente haya que dar la razón a quienes opinan que la religión ha surgido como un subproducto de partes de nuestro cerebro que tuvieron una utilidad más inmediata para nosotros, como la hiperdetección de agentes en la naturaleza comentada anteriormente, pero eso no invalida el hecho de que la conducta religiosa haya acabado mostrando una ventaja adaptativa por sí misma, como han propuesto otros investigadores darwinistas, como más adelante se verá. Precisamente, enlazando con el término 'utilidad', es su tergiversación la que ha llevado a algunos autores a pensar que la religión no es adaptativa. Es el caso de los investigadores que la han tratado como un parásito memético (2) y como un anacronismo (3).

\subsection{La religión como fenómeno no adaptativo: memética y anacronismo}

El principal exponente de la hipótesis memética (2) es R. Dawkins. En su obra El gen egoísta ${ }^{17}$ de gran éxito e igual polémica, este autor emplea la analogía gen-meme. Mientras la perspectiva del gen egoísta entiende al cuerpo como un vehículo para la transmisión de genes, la memética considera a la mente como el vehículo de los memes, es decir, de las unidades mínimas de información cultural. Los memes del mismo modo pueden sobrevivir -entiéndase la metáfora animada-y replicarse vertical y horizontalmente de generación en generación y de individuo en individuo. Las ideas, es decir los memes, son egoístas -siga entendiéndose la metáfora animada-. No importa si benefician o no al individuo, lo único que importa es que sigan existiendo, replicándose de mente en mente como si de una canción pegadiza se tratase. Tampoco tienen por qué hacer la vida de su huésped mejor, ni hacerle feliz, simplemente seguir existiendo y reproducirse. Así es como actúan para sus partidarios los memes religiosos. Sin embargo, Dawkins no es el único exponente de la propuesta memética. Otro de los autores es D. C. Dennett, ${ }^{18}$ quien distingue entre memes salvajes y memes domesticados. Existirían memes salvajes que se habrían generado y evolucionado por encima de las intenciones adaptativas de los individuos y grupos, transmitiéndose vertical y horizontalmente como propone Dawkins. Sin embargo, Dennett afirma que otros sí habrían sido domesticados para proveer beneficios individuales y grupales.

Por su parte, los anacronistas (3), que están estrechamente relacionados con los memeticistas hasta el punto de que en ocasiones los partidarios de unas y otras corrientes interpretativas usan ambas hipótesis complementándolas, focalizan la atención en la disfuncionalidad que presentan las religiones modernas. ${ }^{19}$ Para explicar en qué consistiría este anacronismo, Dennett hace una analogía con nuestra atracción actual por alimentos altamente calóricos a la que denomina sweet tooth. Según este autor, dado que la vida de las tribus cazadoras-recolectoras que nos precedieron era escasa en alimentos, éstas habrían desarrollado el instinto de comer alimentos altamente calóricos para hacer frente a las tareas cotidianas, generándose una necesidad genéticamente innata a este tipo de alimentos como mecanismo de

\footnotetext{
Dawkins 2008 [1976].

Dennet 1998; 1999; 2007.

Dennett 2007.
} 
adaptación. Sin embargo, ese instinto en un contexto predominantemente sedentario como el de las sociedades occidentales actuales ha acabado produciendo un anacronismo que hace que tendamos a preferir los lugares de comida rápida y aumente el índice de obesidad.

Del mismo modo, la religión es vista por este tipo de investigadores como una adaptación ancestral que actualmente es maladaptativa, lo que la habría convertido en un anacronismo frente al pensamiento científico. ${ }^{20}$ Ejemplo de ello serían los tabúes alimenticios producidos por problemas de seguridad como el caso del cerdo y su asociación a la triquinosis. Dicho tabú, antiguamente adaptativo, sería una cuestión anacrónica en el actual contexto occidental. En contra, algunos autores han apuntado las correlaciones existentes entre religiosidad y salud física, lo que en opinión de estos investigadores habría aumentado las probabilidades de sobrevivir y de éxito reproductivo reduciendo la ansiedad y el estrés, promoviendo la cooperación, incrementando la solidaridad de grupo y disminuyendo los conflictos intragrupales.

Vistas en perspectiva ambas hipótesis, mientras la memética ha tratado de explicar la religión como una competición intermemética independientemente del éxito evolutivo individual o grupal de los humanos, la propuesta anacronista resalta que los cambios de contexto se producen más rápido que las adaptaciones, por lo que la religión se encontraría en estos momentos en un cambio de contexto en el que estaría siendo absorbida por otras posturas con mayor éxito evolutivo, caso del pensamiento científico.

Por otro lado, tanto memeticistas como anacronistas todavía no han conseguido llevar a la práctica sus teorizaciones ${ }^{21}$ así como malinterpretan el concepto de éxito evolutivo. ${ }^{22}$ El éxito evolutivo en términos darwinistas se mide en el número de descendencia que se deja generación tras generación. ${ }^{23}$ Es decir, que si tener conductas religiosas hace que se tengan más hijos que potencialmente pueden ser a su vez religiosos frente a quienes no practiquen dichas conductas, la religión habrá resultado un éxito evolutivo. Independientemente de que lo que dicha religión promulgue sea verdad o no, o pretenda engañar a las masas en su propio beneficio, o trate de mantener el orden social establecido, o resulte conveniente con el ideal moral o político que cada uno tenga del mundo, etc. Todas estas cuestiones son teleológicas y secundarias en los términos que aquí se plantean.

\subsection{La religión como fenómeno adaptativo: individual y multinivel}

Por último, quienes postulan que la religión es un fenómeno adaptativo han dividido sus interpretaciones en dos bandos. Por una parte, se encuentran quienes consideran que sólo lo es a nivel individual (4), mientras que por otro lado están quienes lo analizan desde una escala multinivel (5), lo que supone combinar el nivel individual con el nivel de grupo.

\footnotetext{
Smith y Arrow 2010, 59-60.

Aunger 2000.

Smith y Arrow 2010, 57-59.

Un memeticista que parece haber entendido esta idea es el psicólogo D. Campbell (1975), para quien los memes religiosos habrían entrado en competición con otros no religiosos, habiendo salido ganando las conductas que dejan mayor tasa de reproducción para sus seguidores, sean éstas religiosas o no.
} 
Los partidarios de la hipótesis a nivel individual (4) han esgrimido interpretaciones de carácter cognitivo basadas en las potencialidades que la selección natural ha conferido al funcionamiento cerebral, lo que les hace enlazar con los estudios de psicólogos y neurobiólogos darwinistas. Sin embargo, independientemente de que la religión haya surgido como un subproducto, este comportamiento beneficia la adaptación, como se explicaba anteriormente en las críticas a la hipótesis neutra (1). Quienes están a favor de esta hipótesis a nivel individual argumentan que la religión es una adaptación que permite incrementar la cooperación de grupo y los comportamientos altruistas entre individuos sin parentesco. Así, los individuos que mostrasen conductas religiosas serían más ayudados por sus congéneres que si no lo hicieran.

Por su parte, los estudios que han relacionado religión y selección multinivel (5) se han dividido en cuatro interpretaciones.

La primera de ellas es la commitment theory (5.1), comenzada por R. H. Frank ${ }^{24}$ y retomada principalmente por W. Irons ${ }^{25}$ y R. Sosis. ${ }^{26}$ Para estos autores, la religión tiene una base racional y una irracional. Es racional porque se basa en la colaboración del grupo, pero es irracional porque obliga a creer en fuerzas superiores invisibles, ${ }^{27}$ siendo la teoría de juegos la mejor estrategia para valorar las 'pérdidas' y 'ganancias' de los individuos al tomar sus decisiones de cara a ésta. Sin embargo, Sosis era consciente de que durante muchos años no habían existido estudios que aportasen datos a favor de esta hipótesis, ${ }^{28}$ motivo por el cual en dicho artículo realizó un análisis del posible carácter adaptativo de las comunidades religiosas. En él, a partir de una base de datos con 277 comunidades religiosas fundadas entre 1663 y 1937 elaborada por Oved, ${ }^{29}$ Sosis comparó las comunidades religiosas del siglo XIX y principios del XX con otras de carácter laico. La conclusión a la que llegó es que las comunidades laicas son cuatro veces más proclives a desaparecer que las religiosas (fig. 1). El motivo de la continuidad de la comunidad religiosa radica en la fe de sus participantes en que los ideales que promueven y los beneficios que obtienen siendo miembros de ellas es más positivo que en el caso de las laicas, donde la convicción de sus integrantes desaparece más rápido. A esto se une que los grupos religiosos que imponen más requisitos a sus fieles son más longevos que aquéllos que son más laxos a la hora de aceptar fieles, lo que demuestra hasta qué punto las religiones más inflexibles son más exitosas que el resto (fig. 2).

Del mismo modo, Sosis ha extendido sus estudios a los famosos kibbutzim israelíes. Los kibutz, que en hebreo significa agrupación, son comunas agrícolas fundadas por la necesidad de mantener su modo de vida frente a las prácticas agrícolas individuales que dejaron de ser rentables en la práctica. Una de las principales características de estas asociaciones es que la propiedad es colectiva, por lo que los fondos económicos también lo son. El análisis de Sosis ha demostrado que a pesar de que los kibutz religiosos son escasísimos frente a los seculares, desde sus fundaciones no sólo no han tenido los problemas económicos que muestran estos últimos, sino que han tenido más beneficios económicos todos los años que los no religio-

\footnotetext{
Frank 1988.

Irons 1996.

Sosis 2004.

Dow 2006, 70.

Sosis 2000, 73 .

Oved 1988.
} 

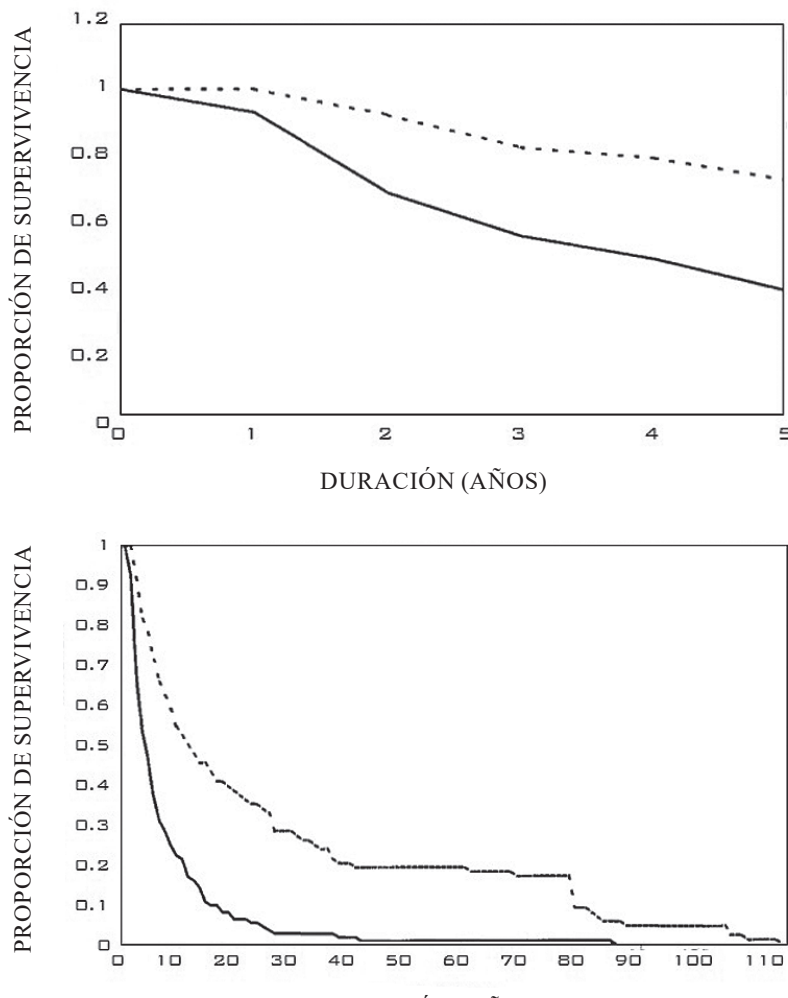

DURACIÓN (AÑOS)

Figura 1. Gráficas comparativas que muestran la duración y el número de comunidades laicas y religiosas en Estados Unidos durante el siglo XIX y principios del siglo XX.

sos. ${ }^{30}$ Una cuestión que surge de estas interpretaciones es porqué los miembros de las comunidades religiosas invierten grandes cantidades de tiempo realizando rituales que desde el punto de vista del fitness evolutivo son tan costosos.

El propio Irons ha postulado que estas señales tienen su razón de ser precisamente en lo costosas que son. Al ser tan difíciles de imitar por miembros que realmente no comulgan con las ideas que promueven estos grupos, es muy difícil que el grupo invierta su tiempo y su esfuerzo en ayudar a personas externas al colectivo. Así, rezar cinco veces al día, lacerarse en penitencia y un largo etcétera de prácticas a veces incomprensibles para quienes las ven desde fuera son signos difícilmente imitables -hard-to-fake signs en la terminología inglesa-. Son conductas costosas desde fuera del grupo, pero rentables para mantener la cohesión del colectivo desde dentro. Visto así, los costosos y extravagantes rituales no son pérdidas de tiempo y esfuerzo, en contra de los presupuestos que fomenta la selección natural, sino prácticas coherentes intra-grupo para mantener su solidaridad y por tanto su perduración en el tiempo. ${ }^{31}$

30 Sosis y Ruffle 2003; Sosis 2004. El mismo resultado ha sido publicado en contextos brasileños (Soler 2008) y neozelandeses (Bulbulia y Mahoney 2008).

31 Sosis y Bressler 2003. 

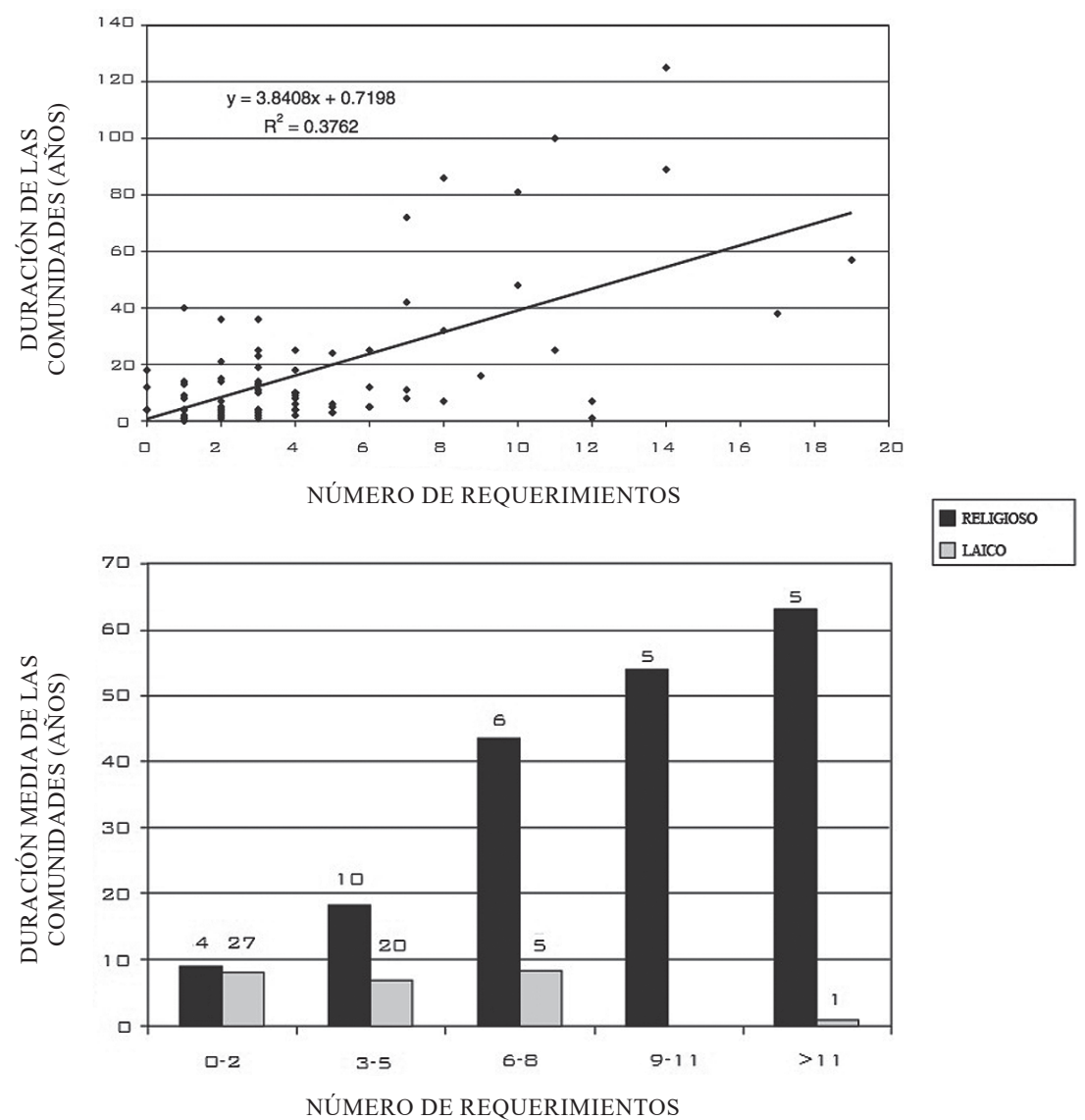

Figura 2. Gráficas comparativas que muestran la duración de las comunidades en años y su relación con el número de requerimientos que pide su organización interna.

En la misma línea hay que citar las ideas publicadas por D. S. Wilson, ${ }^{32}$ seguidas con posterioridad de manera parcial por otros autores. ${ }^{33}$ Para este autor, cada religión ha de ser entendida como un superorganismo en competición constante frente a otros credos rivales. En este sentido, el análisis de cada tradición religiosa habría que realizarlo del mismo modo en que son estudiadas diferentes especies animales compitiendo dentro de un mismo nicho ecológico. Por otro lado, dentro de cada grupo religioso existirían determinadas prácticas rituales que reforzarían la colaboración intra-grupo entre sus fieles. Con el fin de defender estas hipótesis, D. S. Wilson ofrece un variado conjunto de casos que le llevan a analizar en su obra desde el calvinismo al cristianismo primitivo, pasando por el judaísmo y determinadas prácticas religiosas balinesas. ${ }^{34}$ 
De manera paralela y complementando a las anteriores hipótesis se encuentra la propuesta del sociólogo L. Iannaccone. ${ }^{35} \mathrm{Su}$ modelo del comportamiento religioso racional se basa en maximizar los beneficios de pertenecer a un grupo religioso, lo que se consigue cerrando al grupo de agentes externos que puedan desestabilizarlo. Esa sería por ejemplo la misión de los tabúes, impedir que el grupo se diluya en el conjunto de la sociedad cohesionándolo. Dicha cohesión fomenta la solidaridad y viceversa. Los parámetros de lo que es racional o irracional -elementos contraintuitivos- deben de ser medidos desde dentro de la comunidad y no desde fuera, y dicha medición ha de basarse en los beneficios que sacan los miembros del grupo al seguir las imposiciones del mismo.

Una segunda vía es la hipótesis de la regulación ecológica (5.2). ${ }^{36}$ Es la más antigua de todas las propuestas y no empezó desarrollándose en el seno de los estudios darwinistas. Esta alternativa considera que la religión es la manera que tienen los grupos de mandarse señales para adaptarse a los cambios que ocurren en el nicho ecológico en el que se encuentran, evitándose de esta manera que las comunidades se disgreguen con mayor rapidez. M. Harris ha sido uno de sus primeros exponentes desde el materialismo cultural con los conocidos casos del papel sagrado que desempeñan las vacas en la sociedad india o el tabú alimenticio del cerdo en las comunidades judías y musulmanas. ${ }^{37}$ La otra gran figura ha sido R. Rappaport, quien desde posturas funcionalistas desarrolló la idea de que los rituales son señales de grupo que demuestran la necesidad de la religión de actualizarse al contexto cambiante en que se encuentra o no según las necesidades de dicho contexto. Se trataría así de una manera de expresión pública, un barómetro intra-grupo y extra-grupo. Sin lugar a dudas su análisis más notable y conocido fue el del ritual Maring, en el cual se realiza una festividad donde se sacrifican cerdos entre comunidades vecinas, observando las presiones del entorno y de la propia comunidad que conducían a la necesidad de rebajar el número de cerdos con los que convivían para evitar desregulaciones en el seno de la misma. ${ }^{38}$

Autores como Harris o Rappaport han profundizado en la religión como motor del discurso grupo-nicho ecológico, mientras que el darwinismo ha ido un paso más allá y ha introducido la genética y el 'cerebro parcialmente preconfigurado' en esta cuestión. Asunto este último en parte tratado por los estructuralistas y retomado por el contextualismo arqueológico en las últimas décadas.

La tercera propuesta que trata de dar explicación a la religión a nivel de grupo es la hipótesis cultural de grupo (5.3). Bajo este nombre se definen aquellos autores que ponen mayor énfasis, no tanto en lo adaptativas que puedan ser las costumbres religiosas de una sociedad dentro de su nicho ecológico, sino en el hecho en sí de que para los individuos, y por tanto para la sociedad, es más adaptativo seguir las tradiciones culturales heredadas con el paso de las generaciones. Para algunos de estos autores, esto explicaría la cohesión de grupos nómadas incluso teniendo que cambiar estacionalmente de tierras. Un estudio dirigido en esta línea es el desarrollado por Lambert a propósito de la relación proporcional entre el carácter severo recibido por los niños de un total de sesenta y dos culturas prealfabetizadas y la

\footnotetext{
Iannaccone 1992 .

Dow 2006, 74-78.

Harris 2014 [1980].

Rappaport 1987.
} 
severidad de sus dioses. Así, se destaca que cuanto más benévolos son los padres, más benévolos son considerados los dioses y por extensión más sacrificios rituales reciben. ${ }^{39}$

La cuarta hipótesis ha sido desarrollada por J. Diamond, para quien la religión habría sido una estrategia intra-grupo con la que construir una ideología que justificase la cleptocracia (5.4) de una élite a partir de lazos clientelares y nepotistas como manera de conseguir el apoyo popular para preservar la jerarquización social existente. ${ }^{40}$ A pesar del tratamiento peyorativo que hace el autor de esta cuestión, esta idea podría llevar aparejada o no un beneficio del fitness del total de la población derivado de una mayor cohesión social del grupo, por lo que habría que hacer un análisis caso por caso.

\section{Análisis crítico a modo de conclusión}

En estas líneas se ha pretendido analizar cómo se da explicación desde una perspectiva darwinista a la conducta religiosa. En concreto, la religión ha sido vista por los autores darwinistas como un fenómeno adaptativo, negativo o neutro. En muchas ocasiones estas ideas han sido vistas como contrapuestas. Sin embargo, aquí se ha llamado la atención sobre la complementariedad de muchas de ellas. Cuando se ha defendido lo contrario, se han realizado preguntas erróneas que han emborronado el panorama investigativo.

En primer lugar, no existe una 'necesidad de la religión'. Desde un punto de vista exaptativo, para los darwinistas la religión se ha venido apoyando en aptitudes genéticas que fueron la respuesta adaptativa a otras necesidades. En este sentido, el pensamiento darwinista ha venido postulando que, aunque la religión es una exclusividad humana, las bases de las creencias sobrenaturales pueden rastrearse en aspectos como la pareidolia y la hiperdetección de agentes en la naturaleza como mecanismos a través de los cuales se personifican y vivifican fenómenos ocurridos en la naturaleza. Ambas ideas permiten generar creencias en seres superiores. Dichas creencias pueden igualmente pasar desde el punto de vista individual al grupal a través de diversos mecanismos explicados mediante la teoría de juegos para generar actitudes rituales prorreligiosas.

El éxito evolutivo de la antropomorfización de la naturaleza es explicable desde la teoría de juegos, ${ }^{41}$ herramienta metodológica indispensable desde mediados del siglo XX para enlazar la suma de comportamientos individuales a nivel de grupo a partir de la cooperación y la competencia en áreas como la biología o la economía, pero que ha contado con escasísima repercusión en el campo de las humanidades. ${ }^{42}$ Tratando de sintetizar sus resultados, podría decirse que creer en agentes sobrenaturales que pueden sancionar a quienes no cumplan su voluntad tiene a priori escasos riesgos y grandes beneficios, de ahí que se recurra en momentos de ansiedad a la intermediación a seres divinos. ${ }^{43}$

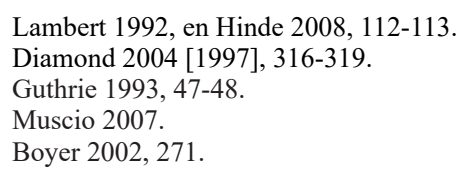


Asumir esta creencia suele generar una tendencia, tanto a nivel individual como social, hacia la realización rituales como hasta ahora se han venido haciendo por si acaso al dejar de hacerlo se generan consecuencias negativas. Es lo que se conoce en teoría de juegos como Apuesta de Pascal, en homenaje al argumento creado por el matemático y filósofo francés Blaise Pascal en 1670. Se trata del miedo a lo desconocido. De seguir la corriente. Esta hipótesis podría explicar parcialmente porqué se perpetúan los ritos. El hecho de que la conducta religiosa surgiera como un subproducto de otras necesidades adaptativas previas no implica que con el paso del tiempo, dichos pensamientos y prácticas religiosas no hayan derivado en conductas positiva o negativamente adaptativas. Es en este punto donde entra en escena el resto de hipótesis.

Entre quienes no ven la religión como un fenómeno adaptativo se ha considerado que este tipo de conducta es una práctica anacrónica, desatendiendo el concepto de adaptación darwinista y focalizando el asunto en la memética como instrumento teórico. No hay que perder de vista nunca la perspectiva de que la adaptación, tanto si es positiva como si es negativa o neutra, debe medirse en términos de fitness evolutivo, es decir en el número de individuos que continúan con las pautas de conducta, en este caso religiosas, de sus predecesores.

Por el contrario, desde la perspectiva adaptacionista toda conducta es adaptativa siempre y cuando quienes lo usen dejen mayor descendencia genética/memética que otros individuos que no lo hagan. Esta es la crítica que han planteado autores como Sosis a los investigadores que han defendido hipótesis anacronistas a propósito de la idoneidad o no de tener creencias religiosas. El propio Sosis ha realizado estudios en la línea opuesta tratando de demostrar que los grupos religiosos son más duraderos que los laicos.

A propósito de esta cuestión, en 2012, H. Rosling fue invitado a participar en las prestigiosas conferencias TED, celebradas ese año en Qatar. ${ }^{44}$ El motivo de su conferencia era mostrar que no existe ninguna relación entre las tasas de natalidad y la religión, para lo cual mostró la evolución de la natalidad desde 1960 hasta ese mismo año. Los datos aportados reflejaron un cambio en la tendencia en la estrategia reproductora de todos los países con censo público en los que se conoce grosso modo la religión de la mayoría de su población. Dicha tendencia ha evolucionado en los últimos 50 años de países con escasa renta per cápita y alto índice de natalidad a países con rentas per cápita cada vez más altas y más bajo índices de natalidad.

De ningún modo esto es una novedad que revoluciona conceptos teóricos. Se trata de un ejemplo más de las famosas estrategias reproductoras $\mathrm{K}$ y $\mathrm{r}$ que presentan todos los seres vivos. O se tiene mucha descendencia sobre la que los cuidados parentales son menores o se tiene poca pero invirtiendo más recursos en sacarla adelante. Debido a la estrecha relación entre la renta per cápita y el índice de natalidad que muestra la misma tendencia en todos los países con independencia de la religión que se profese mayoritariamente en ella, Rosling concluye que la religión no tiene nada que ver en este asunto. En efecto podría dársele la razón a propósito de que las grandes religiones contempladas en su estudio no presentan diferencias sustanciales como para jugar un papel destacable en la natalidad frente al resto de credos reli44 www.ted.com/talks/hans_rosling_religions_and_babies?language=es [Web consultada por última vez el 11 de
marzo de 2017]. 
giosos. Sin embargo, en ningún momento tiene en cuenta si las familias religiosas presentan más altos índices de natalidad que las familias que se declaran no practicantes. En ello consistió el estudio realizado por M. Blume ${ }^{45}$ en el que se concluía que las mujeres religiosas, con independencia de qué credo siguiesen, tenían más altos niveles de descendencia que las mujeres no adscritas a ninguna religión.

En resumidas cuentas, para los darwinistas la religión se presenta como una singularidad humana que hunde sus raíces en características cognitivas que han permitido traducir en clave religiosa las conductas éticas y morales que se comparten con otros primates. El éxito evolutivo de tener una conducta religiosa radica en la minimización de incertidumbres y el reforzamiento de los códigos de comportamiento dentro del grupo, lo que le cohesiona y hace aumentar las probabilidades de supervivencia frente a otras comunidades. En este sentido, algunos psicólogos evolucionistas, neurobiólogos y antropólogos darwinistas han tratado de analizar el porqué de la religión desde diferentes puntos de vista. Sin embargo, no ha sido hasta mediados de la pasada década cuando el movimiento ha tomado conciencia de su multidisciplinariedad en el encuentro que tuvo lugar en la hawaiana isla de Oahu a principios de 2007 con unas jornadas que acabaron dando lugar a la edición de The Evolution of Religion: Studies, Theories, \& Critiques, ${ }^{46}$ seguida de la posterior The Biological Evolution of Religious Mind and Behavior. ${ }^{47}$ En definitiva, tal y como ha expresado Burkert hace escasos años:

Si bien la naturaleza ha dejado de existir como una esencia o una matriz inmutable y aparece más bien [como] un proceso irreversible de autoorganización en patrones transitorios que emergen del caos, no podemos escapar de la participación en ese proceso porque también nosotros hemos sido formados por la antiquísima evolución de la vida. En este sentido, la «naturaleza» biológica está actuando en cada uno de nuestros actos y nuestros pensamientos, igual que los cambios en la naturaleza y las amenazas a las que ésta se ve sometida están afectando nuestra propia existencia. El estudio de la naturaleza y el autoconocimiento humano ya no deberían estar separados, a pesar de que Sócrates, hace mucho tiempo, insistió en que eso era lo correcto. Y si la religión constituye un elemento integrante del mundo humano, que es parte de la naturaleza, comprender la religión debería ser parte del mismo esfuerzo teórico, en un marco de antropología natural (biológica). ${ }^{48}$

\section{Bibliografía}

J. Alvar, «La alquimia del alimento: el sacrificio ritual», ARYS 9 (2011) 21-32.

G. Andrade, El darwinismo y la religión, Universidad de Cantabria, Santander, 2009.

S. Atran, In Gods we trust: the evolutionary landscape of religion, Oxford University Press, New York - Oxford, 2004.

R. Aunger, «Introduction», R. Aunger (ed.), Darwinizing culture. The status of memetics as a science, Oxford University Press, Oxford, 2000, 1-23.

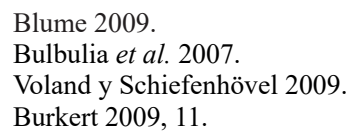


J. Barrett - M. Nyhof, «Spreading nonnatural concepts», Journal of Cognition and Culture 1 (2001) 69-100.

M. Blume, «The Reproductive Benefits of Religious Affiliation», E. Voland y W. Schiefenhövel (eds.), The Biological Evolution of Religious Mind and Behavior. Springer-Verlag, Berlin-Heidelberg, 2009, 117-126.

P. Bourrat, «Origins and Evolution of Religion from a Darwinian Point of View: Synthesis of Different Theories», T. Heams, P. Huneman, G. Lecointre y M. Silberstein (eds.), Handbook of Evolutionary Thinking in the Sciences, Springer, New York, 2015, 761-780.

P. Boyer, Religion Explained. The Human Instincts that Fashion Gods, Spirits and Ancestors, Basic Books, London, 2002.

J. Bulbulia y M. Frean, «Religion as superorganism: on David Sloan Wilson (2002)», M. Stausberg (ed.), Contemporary Theories of Religion. A Critical companion, Routledge, London-New York, 2009, 173-194.

J. Bulbulia y A. Mahoney, «Religious Solidarity: The Hand Grenade Experiment», Journal of Cognition and Culture 8 (2008) 295-320.

J. Bulbulia, R. Sosis, E. Harris, R. Genet, C. Genet y K. Wyman, The Evolution of Religion: Studies, Theories \& Critiques, Collins Foundation Press, Santa Margarita, 2007.

W. Burkert, La creación de lo sagrado. La huella de la biología en las religiones antiguas, Acantilado, Barcelona, 2009.

D. Campbell, «On the conflicts between biological and social evolution and between psychology and moral tradition», American Psychologist 30 (1975) 1103- 1126.

R. Dawkins, El gen egoísta, Salvat, Barcelona, 2008 (1976).

D. C. Dennett, «The Evolution of Religious Memes: Who or What Benefits?», Method \& Theory in the Study of Religion 10 (1998) 115-128.

D. C. Dennett, La peligrosa idea de Darwin. Evolución y significados de la vida, Galaxia Gutenberg, Barcelona, 1999.

D. C. Dennett, Romper el hechizo. La religión como fenómeno natural, Katz Buenos Aires, 2007.

J. Diamond, Armas, gérmenes y acero. Breve historia de la humanidad en los últimos trece mil años, Debate, Barcelona, 2004 (1997).

J. W. Dow, «The evolution of religion: three anthropological approaches», Method and Theory in the Study of Religion 18 (2006) 67-91.

R. H. Frank, Passions Within Reason: The Strategic Role of the Emotions, Norton, New York, 1988.

S. J. Gould, La estructura de la teoría de la evolución, Tusquets Editores, Barcelona, 2004.

S. Guthrie, «A cognitive theory of religion», Current Anthropology 21: 2 (1980) 181-194.

S. Guthrie, Faces in the clouds. A new theory of religion, Oxford University Press, Oxford, 1993.

G. Gutiérrez, «Evolución biológica», J. L. Escacena, D. García, F. J. García (eds.), Clasificación y arqueología. Enfoques y métodos taxonómicos a la luz de la evolución darwiniana, Universidad de Sevilla, Sevilla, 2010, 15-31.

M. Harris, Vacas, cerdos, guerras y brujas, Alianza Editorial, Madrid, 2014 (1980).

R. A. Hinde, ¿Por qué persisten los dioses? Una aproximación científica a la religión, Intervención Cultural, Barcelona, 2008.

L. Iannaccone, «Sacrifice and Stigma: Reducing Free-Riding in Cults, Communes, and Other Collectives», Journal of Political Economy 100 (1992) 271-292.

W. Irons, «Morality, Religion, and Human Evolution», W. M. Richardson y W. J. Wildman (eds.), Religion and Science: History, Method, Dialogue, Routledge, New York, 1996, 375-399. 
L. Kirkpatrick, Attachment, evolution, and the psychology of religion, Guilford Press, New York, 2005.

E. Mayr, Una larga controversia. Darwin y el darwinismo, Crítica, Barcelona, 1992.

H. Muscio, «Sociabilidad y mutualismo durante las expansiones agrícolas en entornos fluctuantes: un modelo de teoría evolutiva de juegos aplicado al poblamiento del periodo temprano de la Puna de Salta, Argentina», A. E. Nielsen, M. C. Rivolta, V. Seldes, M. M. Vázquez y P. H. Mercolli (comps.), Producción y circulación prehispánicas de bienes en el sur andino, Editorial Brujas, Córdoba, 2007, 105-133.

Y. Oved, Two hundred years of American communes, Transaction Publishers, New Brunswick, 1988.

L.-G. Pérez-Aguilar, «Evolucionismos y ciencias históricas: darwinismo vs. lamarckismo en arqueología», Spal 20 (2011) 23-41.

I. Pyysiainen, M. Lindeman y T. Honkela, «Counterintuitiveness as the hallmark of religiosity», Religion 33: 4 (2003) 341-355.

R. A. Rappaport, Cerdos para los antepasados. El ritual en la ecología de un pueblo en Nueva Guinea, Siglo XXI, Madrid, 1987.

M. Ruse, Charles Darwin, Katz, Madrid, 2008.

Z. Smith y H. Arrow, «Evolutionary Perspectives on Religion: An Overview and Synthesis», EvoS Journal: The Journal of the Evolutionary Studies Consortium 2: 2 (2010) 48-66.

M. Soler, «Commitment Costs and Cooperation: Evidence from Candomble, and Afro-Brazilian Religion», J. Bulbulia, R. Sosis, E. Harris, R. Genet, C. Genet y K. Wyman (eds.), The Evolution of Religion: Studies, Theories, and Critiques, Collins Foundation Press, Santa Margarita, 2008, 167-174.

R. Sosis, «Religion and intragroup cooperation: preliminary results of a comparative analysis of utopian communities», Cross-Cultural Research 34: 1 (2000) 70-87.

R. Sosis, «The Adaptative Value of Religious Ritual», American Scientist 92: 2 (2004) 166172.

R. Sosis, E. R. Bressler, «Cooperation and commune longevity: a test of the costly signaling theory of religion», Cross-Cultural Research 37: 2 (2003) 211-239.

R. Sosis y B. J. Ruffle, «Religious ritual and cooperation: testing for a relationship on israeli religious and secular kibbutzim», Current Anthropology 44 (2003) 713-722.

E. Voland y W. Schiefenhövel, (eds.), The Biological Evolution of Religious Mind and Behavior, Springer, Berlin - Heidelberg, 2009.

E. O. Wilson, Sobre la naturaleza humana, Fondo de Cultura Económica, México D. F., 1980.

D. S. Wilson, Darwin's Cathedral. Evolution, Religion, and the Nature of Society, The University of Chicago Press, Chicago, 2002. 